\title{
TAGUNG
}

\section{Jahre Römische Verträge: Rückblick und Perspektiven}

\author{
Peter Kuffel, Dominic Maugeais und Eike Zaumseil*
}

Mit der Einigung auf ein Reformmandat am 21./22. Juni 2007 beendeten die Staats- und Regierungschefs der 27 Mitgliedstaaten der Europäischen Union die seit dem Scheitern der Referenden in Frankreich und den Niederlanden andauernde Phase des Stillstands des Integrationsprozesses. Vor diesem Hintergrund fand auf Einladung des Instituts für Europäische Politik (IEP) in Zusammenarbeit mit dem Arbeitskreis Europäische Integration (AEI) und dem Centre International de Formation Européenne (CIFE) im Jean-MonnetHaus in Berlin eine wissenschaftliche Konferenz statt. Ziel dieser interdisziplinären Tagung war es, die fünfzigjährige europäische Integrationsgeschichte seit den Römischen Verträgen zu beleuchten und Perspektiven für die Zukunft der Europäischen Union zu erörtern. Auf der interdisziplinär ausgerichteten Veranstaltung sprachen Geschichts-, Politik-, Wirtschafts- und Rechtswissenschaftler/innen sowie Abgeordnete des Europäischen Parlaments und Vertreter europäischer Forschungsinstitutionen.

Die Zukunft Europas in historischer Perspektive

Im Zentrum der Eröffnungsbeiträge stand der Blick in die Vergangenheit, um die ,historische Zukunft ' Europas zu beleuchten und Perspektiven für die Zukunft zu entwickeln. Für Jürgen Kocka sind die wichtigsten Motive für die europäische Integration - die Kriegserfahrung und die Ost-West-Konfrontation - nicht mehr gegeben. Auf der Suche nach anderen Antriebskräften fragte Kocka nach den langfristigen historischen Bedingungen europäischer Identitätsbildung. Er verortete identi-

\section{Jahre Römische Verträge: eine rückblickende Bewertung des Integrationsprozesses und Ausblick auf die Zukunft Europas}

Wissenschaftliche Konferenz des Instituts für Europäische Politik (IEP) in Zusammenarbeit mit dem Arbeitskreis Europäische Integration

(AEI) und dem Centre International de

Formation Européenne (CIFE) mit freundlicher Unterstützung des Auswärtigen Amtes und der Europäischen Kommission

Berlin, 28./29. Juni 2007

\section{Begrüßung und Eröffnung}

Prof. Dr. Peter-Christian MÜLLER-GRAFF, Direktor des Instituts für deutsches und europäisches Gesellschafts- und Wirtschaftsrecht, Universität Heidelberg, Vorstandsvorsitzender des AEI, Heidelberg/Berlin

Dr. Barbara LIPPERT, Stellvertretende Direktorin des Instituts für Europäische Politik (IEP), Berlin

\section{Die historische Zukunft Europas}

Vorsitz

Prof. Dr. Siegfried MAGIERA, Lehrstuhl für öffentliches Recht, insbesondere Völker- und Europarecht/Jean-Monnet-Lehrstuhl für Europarecht, Speyer

\section{Einführungen}

Prof. Dr. Jürgen KOCKA, Lehrstuhl für Geschichte der industriellen Welt, Freie Universität Berlin

Prof. Wilfried LOTH, Lehrstuhl für Neuere und Neuste Geschichte, Universität Duisburg-Essen

Prof. Dr. Wolfgang SCHMALE, Lehrstuhl für Neuere und Neuste Geschichte, Universität Wien

* Peter Kuffel, Institut für Europäische Politik, Berlin. Dominic Maugeais, Institut für Europäische Politik, Berlin. Eike Zaumseil, Institut für Europäische Politik, Berlin. 
tätsstiftende Momente im Spannungsfeld zwischen der Abgrenzung von anderen Kulturen einerseits und der Verflechtung mit anderen Kulturkreisen andererseits. Diesem Umstand müsse durch durchlässige Grenzen als Chance europäischer Identitätsbildung Rechnung getragen werden.

Demgegenüber erläuterte Wilfried Loth die mittelfristige Perspektive der europäischen Integration seit Mitte des 20. Jahrhunderts. Dabei seien vier wesentliche Integrationsmotive identifizierbar und über das Ende des Ost-WestKonfliktes hinaus relevant: die Förderung wirtschaftlicher Produktivität und Stabilität in Europa, die langfristige Friedensicherung, die Stärkung der internationalen Handlungsfähigkeit sowie die Einbindung Deutschlands. Der zunehmende Prozess der Verflechtung erfasse jedoch nicht alle Teile der europäischen Gesellschaften gleichermaßen. Die Herausbildung einer genuin europäischen Öffentlichkeit sei zwar noch nicht erkennbar, es könne jedoch von einer europäischen Identität im Singular gesprochen werden, deren Basis das gemeinsame Verfassungserbe bilde. Die europäische Integration fördere nicht das Absterben der Nationalstaaten und der nationalen Identitäten, sondern ermögliche erst ihr Überleben in abgewandelter Form.

Die Frage nach der Zukunft Europas sei im Wesentlichen eine Frage nach der Zukunft der Europäischen Union, so Wolfgang Schmale. Aus der Perspektive des Historikers stehe das Interesse an den Paradigmen der Integration im Vordergrund. Das Paradigma der Einheit sei zu etwas Heiligem erhoben worden, dies müsse jedoch überdacht werden. Die Union habe sich seit jeher in einem Spannungsverhältnis zwischen Einheit und nationalstaatlicher beziehungsweise regionaler Vielfalt befunden. Das Paradigma des Netzwerkes böte einen Denkrahmen, bei dem nicht mehr die Einheit der Union, sondern die Wahrung der Kohärenz des Systems im Mittelpunkt stehe. Die zukünftigen Debatten werden sich, so Schmale, im Spannungsfeld der Paradigmen der Einheit und des Netzwerkes bewegen.
Epochen der europäischen Integration von den Anfängen bis in die Zukunft

Vorsitz

Prof. Dr. Rudolf HRBEK, Lehrstuhl für Innenund EU-Politik, Universität Tübingen, Präsident des AEI

Wirtschaftliche Entwicklungsphasen

Prof. Dr. Wim KÖSTERS, Lehrstuhl für theoretische Volkswirtschaftslehre, Ruhr-Universität Bochum

Primärrechtliche Entwicklungsphasen

Prof. Dr. Peter-Christian MÜLLER-GRAFF, Universität Heidelberg

Etappen des Erweiterungsprozesses

Dr. Barbara LIPPERT, Stellv. Direktorin, Institut für Europäische Politik, Berlin

Entwicklungseinflüsse der Weltpolitik

Prof. Dr. Werner LINK, Universität zu Köln

Erfahrungen und Wahrnehmungen der Integrationsentwicklung in unterschiedlichen Mitgliedstaaten

Vorsitz

Dr. Hartmut MARHOLD, Generaldirektor, Centre International de Formation Européenne (CIFE), Nizza

\section{Einführungen}

Prof. Dr. Gianni BONVICINI, Direktor, Istituto Affari Internazionali, Rom

Prof. Dr. William E. PATERSON, Direktor, Institute for German Studies, Universität Birmingham

Dr. Alfred PIJPERS, Senior Research Fellow, Institute of International Relations „Clingendael", Den Haag

Dr. Vladimir HANDL, Institut für Internationale Beziehungen, Prag

Dr. Sylvie GOULARD, Präsidentin, Mouvement Européen France, Paris

Nach dem europäischen Juni-Gipfel: Fortsetzung oder Neuanfang des konstitutionellen Reformprozesses?

Vorsitz

Prof. Dr. Mathias JOPP, Direktor, Institut für Europäische Politik, Berlin

Wege zur Rettung des Verfassungsprozesses Prof. Dr. Ingolf PERNICE, Lehrstuhl für öffentliches Recht, Völker- und Europarecht, Humboldt-Universität zu Berlin 


\section{Entwicklungsphasen europäischer Integra- tion}

Im zweiten Teil der Konferenz ging es um eine rückblickende Bilanzierung der Etappen des europäischen Integrationsprozesses aus verschiedenen disziplinären Blickwinkeln.

Nach einem Überblick über die wesentlichen Etappen der Wirtschaftsintegration, ging Wim Kösters der Frage nach, ob Europa mit der Wirtschafts- und Währungsunion im internationalen Vergleich adäquat auf die Herausforderungen der Weltwirtschaft reagiert habe. Kösters präsentierte empirische Befunde dafür, dass das Wirtschaftswachstum in den Staaten der Wirtschafts- und Währungsunion im Durchschnitt hinter anderen Regionen der Welt zurückbleibe. Jedoch divergierten die Entwicklungen in den Mitgliedstaaten. Als Ursache betrachtete Kösters deshalb nicht die Wirtschaftsintegration als solche, sondern die mangelnde Anpassung an die neuen Rahmenbedingungen. Ausbleibende Wohlstandsgewinne könnten längerfristig $\mathrm{zu}$ einem Problem für die Output-Legitimität der Europäischen Union werden.

Mit der Erläuterung der primärrechtlichen Entwicklungsphasen der Gemeinschaftsintegration skizzierte Peter-Christian MüllerGraff die Gestaltung der rechtlichen Dimension der europäischen Integration. Aus rechtlicher Sicht seien vor allem die Marksteine der Herausbildung des historisch neuartigen Primärrechtes durch die Ablösung vom Recht klassischer, völkerrechtlicher Organisationen von Interesse. Als derartige „Mutationen“, so Müller-Graff, seien vor allem zu nennen: die „Übertragung“ von Hoheitsrechten im Zuge der Montanunion, die Etablierung des Prinzips der unmittelbaren Wirkung sowie des Anwendungsvorranges durch den Europäischen Gerichtshof, die Legitimations- und Kompetenzveränderung des Europäischen Parlaments im Zuge des Direktwahlakts von 1976/1978, die Einschränkung des Souveränitätsprinzips durch die qualifizierte Mehrheitsentscheidung, das Aufkommen nicht-marktintegrativer Politikbereiche sowie die
Stufen der konstitutionellen Evolution und Wege aus der Verfassungsfalle

Dr. Andreas MAURER, Leiter der Forschungsgruppe EU-Integration, Stiftung Wissenschaft und Politik, Berlin

Neugründung, Kerneuropa und konzentrische Kreise

Prof. Dr. Klaus HÄNSCH MdEP, Ehem. Präsident des Europäischen Parlaments, Brüssel

Chancen und Risiken des Verfassungsprozesses Dr. Andrew DUFF MdEP, Mitglied des konstitutionellen Ausschusses, Europäisches Parlament, Brüssel

Verankerung der Gleichwertigkeit jedes Unionsbürgers bei qualifizierten Mehrheitsentscheidungen mittels der demografischen Nachkontrollmöglichkeit im Vertrag von Nizza.

Barbara Lipperts Vortrag zu den Etappen des Erweiterungsprozesses orientierte sich an der Erklärung der Dynamik der Erweiterung sowie der Stabilität der Gemeinschaft im Erweiterungsprozess. Vor allem das Selbstverständnis der Gemeinschaft der Sechs als ein nicht abgeschlossenes Projekt habe zur Beitrittsdynamik beigetragen. Nationalstaatliche Funktionsdefizite sowie die Nutzung der Beitrittsperspektive als Modernisierungshebel könnten als wesentliche Motive des Beitrittsersuchens identifiziert werden, so Lippert. Für die Dynamik der Erweiterung in den 1990er Jahren sei entscheidend gewesen, dass sich in Osteuropa keine politische Gemeinschaft herausgebildet habe, die sich als Alternative zur Europäischen Gemeinschaft angeboten hätte. Eine Grundvoraussetzung für die Stabilität des Erweiterungsprozesses, so Lippert, sei die Tragfähigkeit der Kerngruppe um Deutschland und Frankreich gewesen. Nicht zuletzt habe die einheitliche und auf den Acquis eingeschworene Verhandlungsführung der Gemeinschaft sowie die Fortführung der Vertiefung parallel zur Erweiterung die Stabilität des Prozesses gefördert.

In seiner Analyse der externen Entwicklungseinflüsse auf den Verlauf der europäischen Integration betrachtete Werner Link die Ent- 
wicklung der europäischen Integration im Spannungsfeld des Ost-West-Konflikts und in der Zeit danach. Dabei hätten externe Herausforderungen integrationsfördernde Wirkung gehabt, Bindungen an eine externe Großmacht jedoch Integrationsprozesse gehemmt. Die Sowjetunion habe die Rolle des negativen, die USA die des positiven Katalysators gespielt. Gemeinsame Außen- und Sicherheitspolitik und Europäische Sicherheits- und Verteidigungspolitik seien erst nach dem Ende des Kalten Krieges möglich geworden. Einerseits seien die USA zum externen Divisor im europäischen Integrationsprozess geworden, weil die Union nicht mehr geschlossen hinter der Außenpolitik der USA stehen müsse. Andererseits habe die Europäische Union gegenüber Amerika auch gemeinsame Positionen entwickelt. Insgesamt seien externe weltpolitische Einflüsse in beiden Epochen nachweisbar.

Zwischen Ängsten und Skepsis: Die Perzeptionen der Integrationsentwicklung in den Mitgliedstaaten

Wie lässt sich, fragte Gianni Bonvicini, die Enttäuschung der Italiener über die schwindende integrative Kraft der Europäischen Union erklären? Die Europäische Union sei alternativlos für Italien, erläuterte Bonvicini, und erfülle wichtige externe Steuerungs- und Beschränkungsfunktionen für das fragile politische System. Gleichzeitig wolle Italien eine entscheidende Rolle innerhalb der Union spielen, fürchte aber aufgrund seiner wirtschaftlichen Probleme in einer losen Union der konzentrischen Kreise nicht zur Kerngruppe zu gehören. Initiativen außerhalb der Verträge, wie etwa ,Schengen' oder ,Prüm', seien nicht im Sinne der Italiener. Italien hätte den Traum eines föderalen Europas. Eine in weiten Bereichen intergouvernementale Union würde Italien aller Wahrscheinlichkeit nach marginalisieren.

Bei der Darstellung der britischen Perzeption des Integrationsprozesses ging William Paterson vom EG-Beitritt des Vereinigten König- reiches 1973 aus. Der Beitritt habe die Defizite des eigenen Nationalstaats beseitigen sollen. Die im Zuge der Dekolonisierung entstandene konjunkturelle Delle habe die Entscheidung für den Beitritt beschleunigt. Die am Erschließen neuer Absatzmärkte interessierte Regierung Thatcher habe übersehen, welche Effekte die Anwendung der qualifizierten Mehrheit für die britische Politik haben würde. Heute seien die Briten wegen ihrer Präferenz für Deregulierung zu Vernunfteuropäern geworden. Der politische Diskurs sei nach wie vor skeptisch. Die politische Klasse habe zum einen britische Interessen stets so definiert, dass ein fundamentales Interesse an der EU-Mitgliedschaft und der Effektivität der Gemeinschaft nicht sichtbar geworden sei. Ein gesellschaftlicher Konsens in Bezug auf die Mitgliedschaft in der Union sei nie hergestellt worden. Zum anderen existiere ein history gap. Im Vereinigten Königreich tue man sich schwer, mit der neuesten Geschichte des Landes zu argumentieren, man greife stets auf die Ereignisse des Zweiten Weltkrieges zurück.

Alfred Pijpers thematisierte den Wandel der niederländischen EU-Integrationspolitik von ihrer einstigen Vorreiterrolle hin zu einer verhalteneren Position. Zwar gebe es in weiten Teilen der Gesellschaft weiterhin diffuse Unterstützung für die Europäische Union und ihre Institutionen. Der fundamentale Wandel liege jedoch darin, dass der Wille einer weiteren Vertiefung nicht mehr vorhanden sei. Föderale Finalitätsvorstellungen seien hinter die Idee eines bloßen Staatenverbundes zurückgetreten. In den Niederlanden, so Pijpers, sei es zu einer Renationalisierung des politischen Lebens gekommen. Im Zusammenhang mit gravierenden sozialen Problemen, hervorgerufen durch massive Zuwanderung und mangelnde Integrationsmaßnahmen, sei der Bedarf an nationaler Steuerungsfähigkeit gestiegen. Brüssel erscheine vielen als Zentrum, das versucht, den Nationalstaat eben dieser Steuerungsfähigkeit zu berauben, ohne jedoch die gleiche Problemlösungsfähigkeit zu besitzen. 
In Polen und Tschechien werde die Europäische Union grundsätzlich als äußerst attraktiv angesehen, was an drei Punkten festgemacht werden könne, so Vladimir Handl. Erstens werde sie als Friedens-, jedoch nicht als Sicherheitsgemeinschaft wahrgenommen. Zweitens sei sie als Wirtschaftsgemeinschaft eine Lebensstandardgemeinschaft. Drittens sei die Union eine Wertegemeinschaft, die als Rechtsgemeinschaft den Schutz des Individuums garantiere. Insgesamt herrsche ein reduziertes EU-Bild. Im Vordergrund stünden die Leistungen der Union gegenüber ihren Mitgliedern, die Notwendigkeit zu Engagement werde ausgeblendet. Handl erklärte das mit drei Entwicklungen: Zum Ersten fehle es an politischer Nähe der beiden Länder zur Europäischen Union. Das EU-Bild werde für innenpolitische Zwecke verzerrt, wobei auf die Machtverteilung in der Union abgestellt werde. Zum Zweiten sei das Vertrauen in Politik und Institutionen in Polen und Tschechien miserabel und der politischen Klasse fehle die Akzeptanz für die Methode-Monnet, die wegen ihrer Ergebnisoffenheit und Mehrdeutigkeit als Bedrohung gewertet werde. Zum Dritten trügen die Medien zum fragmentierten Europa-Bild bei, indem sie sich nur mit Details der Wirtschaftspolitik befassten.

Für Sylvie Goulard erscheint der Zustand der europäischen Integration paradox. Die Europäische Union ist 50 Jahre alt, werde aber in den Mitgliedstaaten immer weniger als Chance zur Lösung gemeinsamer Herausforderungen begriffen, sondern vielmehr als Bedrohung nationalstaatlicher Kompetenzen. Einerseits wolle man eine Europäische Union, so handlungsfähig und stark wie die USA, anderseits bleibe man dem berühmten „Faire l'Europe, sans défaire la France“" verhaftet. Man müsse jedoch erkennen, so Goulard, dass man den Weg einer föderalen Union schon längst beschritten habe. Es komme darauf an, Kompetenzübertragungen nicht immer nur abzuwehren, sondern der Europäischen Union zu ermöglichen, als einheitlicher Akteur in der Welt aufzutreten.
Vom Verfassungs- zum Reformvertrag: Chancen und Risiken

Der Vortrag von Ingolf Pernice griff die Frage auf, ob der konstitutionelle Reformprozess nun fortgesetzt werde oder vor einem Neuanfang stehe. Im Bereich des Acquis sei der entscheidende Sprung bereits gelungen. Auch wenn der neue Vertrag nicht als Verfassung bezeichnet werde, erfülle er trotzdem die Funktion einer Konstitution und trage zu mehr Ehrlichkeit in der Europäischen Union bei. Für die Zukunft des Reformvertrages bestehe die Gefahr darin, dass einzelne Staaten nun versuchen könnten, vieles neu zu verhandeln. Regierungen müssten sich an das halten, was sie bereits ratifiziert haben. Zur Ratifizierung des Vertrages bedürfe es keiner Volksabstimmungen, denn der komplexe Vertragstext sei nicht referendumsfähig. Eine parlamentarische Ratifizierung genüge in repräsentativen Demokratien. Insgesamt handele es sich sowohl um die Fortsetzung des Konstitutionalisierungsprozesses als auch um einen Neuanfang. Der funktionale Verfassungsbegriff auf EU-Ebene bedeute nicht den völligen Verlust der Souveränität der Nationalstaaten, sondern im Gegenteil die Legitimierung und Begrenzung supranationaler Betätigung.

In eine Falle führten - so Andreas Maurer neuere Trends des Integrationsprozesses: die Differenzierung und Reintergouvernementalisierung. Handlungsermächtigungen für die supranationale Ebene würden durch alternative Methoden verwässert und häufig außerhalb der Verträge geregelt. Des Weiteren werde der Europäische Rat unverhältnismäßig gestärkt. Er habe sich von einem Impulsgeber hin zum Beschlussorgan gewandelt. Neben dem Trend zur Verwässerung des Integrationsprozesses gebe es auch das Phänomen der Diffusion politischer Verantwortung. Hierbei komme es zu einer Gruppenbildung am Rande der europäischen Institutionen. Die Falle bestehe hier darin, dass man sich zwar pro-europäisch geben könne, aber gegen die Vertiefung der Integration sei und umgekehrt. 
Die Lösung liege schlicht darin, vom europäischen Pathos wegzukommen und sich des 50jährigen Integrationsprozesses zu vergegenwärtigen, in dessen Charakter das Besondere des EU-Systems liege: Die Verbindung des Technokratisch-Bürokratischen mit einer supranationalen Form der Demokratie. Die Union sei die Summe aller aufgrund der Verträge errichteten Institutionen. Sie sei ein dynamischer und zieloffener Prozess, woran ein Reformvertrag nichts ändern werde. Im Langzeittrend sei stets sowohl eine Ausdifferenzierung von Verhandlungs- und Entscheidungssystemen als auch eine Demokratisierung zu beobachten gewesen.

Klaus Hänsch betonte, dass der Reformvertrag, gemessen am Zustand, in dem sich die Europäische Union mit den Verträgen von Nizza befinde, als großer Fortschritt bewertet werden könne. Der Reformvertrag verdeutliche jedoch, dass das Ziel einer Europäischen Föderation zur Illusion geworden sei. Eine Neugründung der Union im Fall eines Scheiterns des Reformvertrages sei faktisch wie konzeptionell unmöglich. Das Konzept der konzentrischen Kreise sei nicht überzeugend, da kein Zentrum existiere, um das sich diese Kreise anordnen könnten. Ebenso wenig praktikabel sei das Konzept der zwei Geschwindigkeiten, da es auf der Idee zweier immer gleicher Staatenkonstellationen basiere, die kontinuierlich mit ungleicher Geschwindigkeit voranschreiten würden. Einzig die Idee eines Kerneuropas könne auf der Grundlage der verstärkten Zusammenarbeit innerhalb der Verträge angestrebt werden, wobei ein Gebilde mehrerer kleinerer Kerne entstehen würde. Dabei müsse jedoch sichergestellt werden, dass die Kohärenz der Union gewahrt werde.
Zum Abschluss sprach Andrew Duff über die Chancen und Risiken des europäischen Verfassungsprozesses. Das Jahr 2007 werde als ein Jahr in Erinnerung bleiben, in dem das gegenwärtige Verfahren zur Vertragsreform an seine Grenzen angelangt sei. Integrationswillige Länder in der Europäischen Union müssten ausführlich von der Möglichkeit der, Verstärkten Zusammenarbeit' Gebrauch machen, insbesondere in den Bereichen der ehemals Dritten Säule. Damit werde deutlich, dass neben dem Ziel einer Erleichterung des Vertragsreformverfahrens auch innerhalb des bestehenden vertraglichen Rahmens ein zielstrebiges Voranschreiten der integrationswilligen Staaten angestrebt werde. Weiterhin werde dem Europäischen Parlament durch die Verträge das Recht eingeräumt, Vertragsreformen anzuregen. Es werde in der Lage sein, konstruktive Vorschläge für ein konstitutionelles Arrangement zu unterbreiten.

\section{Schlussbemerkung}

Im Rahmen dieser Tagung wurde die Entwicklung der europäischen Integration aus historischer, rechtlicher, politischer und wirtschaftlicher Sicht beleuchtet und Perspektiven für die Zukunft der Europäischen Union erörtert. Damit ermöglichte die Tagung vielfältige Einblicke in die fünfzigjährige Integrationsgeschichte seit den Römischen Verträgen und bot Gelegenheit zu einer regen interdisziplinären Diskussion, in der eine Vielzahl von kontroversen Fragen angesprochen wurden. Die zeitliche Nähe zum Juni-Gipfel verlieh dem auf der Tagung erzielten Spagat zwischen Rückblick und Ausblick eine besondere Aktualität. 